\title{
Prevención de la bacteriemia relacionada con catéter intravascular
}

\section{Prevention of the intravascular catheter-related bacteremia}

\section{Lorente}

\author{
Unidad de Cuidados Intensivos, Hospital Universitario de Canarias, La Laguna, Tenerife, España \\ Disponible en Internet el 30 de octubre de 2010
}

Los pacientes ingresados en las Unidades de Cuidados Intensivos $(\mathrm{UCl})$ frecuentemente precisan la canalización de un catéter venoso central (CVC) ${ }^{1,2}$. Este procedimiento presenta el riesgo de diferentes complicaciones infecciosas y mecánicas $^{3-6}$, las cuales conllevan un incremento de la morbimortalidad y de los costes asistenciales ${ }^{7-13}$. Por ello, es necesaria la implementación de una serie de medidas para intentar evitar estas complicaciones.

La incidencia de bacteriemia relacionada con catéter venoso central (BCVC) se encontraba alrededor de 5 episodios/1.000 días de CVC a nivel nacional ${ }^{14,15}$, que estaba por encima de la incidencia de 2-3 episodios/1.000 días de CVC en Europa ${ }^{16}$ y Norteamérica ${ }^{17,18}$ y por debajo de la incidencia de 7 episodios/1.000 días de CVC en Latinoamérica y Asia $^{19}$. Por otra parte, la aplicación de un programa para la prevención de BCVC había conseguido disminuir su incidencia en diferentes experiencias, como en la de Provonost en 103 $\mathrm{UCl}$ del estado de Michigan ${ }^{20}$ y en la de Warren en $13 \mathrm{UCl}$ de seis hospitales americanos ${ }^{21}$. Por lo tanto, con el propósito de disminuir la incidencia de BCVC en las UCl españolas, la Sociedad Española de Medicina Intensiva Crítica y Unidades Coronarias con la colaboración de la Alianza Mundial para la Seguridad del Paciente de la Organización Mundial de la Salud y la Agencia de Calidad del Ministerio de Sanidad y Consumo elaboraron el Proyecto «Bacteriemia Zero» ${ }^{22}$. Este proyecto constaba de dos actividades complementarias: 1) implantar un programa de formación y una serie de medidas en relación con la inserción y manejo de los catéteres para disminuir la densidad de incidencia nacional de BCVC por debajo de 4 005

Véase contenido relacionado en DOI: 10.1016/j.medin.2010.07.

Correo electrónico: lorentemartin@msn.com episodios por 1.000 días de CVC, y 2) implantar un plan de seguridad integral para promover la cultura de la seguridad en el trabajo diario. Para la disminución de la BCVC se propusieron las siguientes actividades: programa de formación en la prevención de BCVC, instauración de seis medidas para la prevención de la BCVC (higiene adecuada de manos, uso de medidas de barrera de máxima asepsia durante la inserción del CVC, uso de clorhexidina en la preparación de la piel, preferencia de la vena subclavia como lugar de inserción, retirada de CVC innecesarios y manejo higiénico de los catéteres), lista de comprobación durante la inserción de los CVC y carro exclusivo para los accesos vasculares. Para promover la cultura de la seguridad en el trabajo diario se propusieron las siguientes actividades: lista de objetivos diarios, registro de seguridad, encuesta de seguridad y campaña de manos limpias.

Para valorar la aplicabilidad a nivel nacional del Proyecto Bacteriemia Zero se desarrolló un estudio piloto, el cual es motivo de esta editorial ${ }^{23}$. El estudio fue realizado en $17 \mathrm{UCl}$ españolas de tres comunidades autónomas durante tres meses (1 de octubre al 31 de diciembre de 2007). Con este estudio se concluyó que era viable la aplicación del proyecto a nivel nacional, se identificaron una serie de aspectos mejorables para la implantación a nivel nacional y además se disminuyó la densidad de incidencia de BCVC.

Respecto al cumplimiento de las acciones para la prevención de la bacteriemia se detectaron los siguientes problemas: el carro exclusivo para los accesos vasculares no se creó en ninguno de los hospitales, hubo dificultades para la desinfección con clorhexidina en algunas unidades y no se cuantificó la retirada de catéteres innecesarios.

En relación a la cultura de seguridad, cerca del $80 \%$ respondió a la encuesta de seguridad. Aproximadamente la 
mitad de los encuestados opinaban que era necesario más personal, que el exceso de personal temporal disminuía la seguridad, que no se discuten los errores, que no existe apoyo por parte de superiores y dirección/gerencia y que no existe cooperación entre los servicios hospitalarios. Ademâs, se apreciaron importantes deficiencias en el registro de problemas relacionados con el uso de CVC, la campaña de manos limpias y la lista de objetivos diarios.

En lo que concierne a la bacteriemia, en las $\mathrm{UCI}$ del grupo intervención disminuyó la densidad de incidencia comparada con los años 2004-2006 de 6,06-7,54 a 3,85 episodios/1.000 días de CVC $(p=0,003)$. Pero también disminuyó en las UCI del grupo control de 12,31-14,40 a 3,31 episodios/1.000 días de CVC $(p<0,001)$. Estos resultados respecto a la disminución de la BCVC son interesantes, pero el estudio presenta algunas limitaciones como son las siguientes: la selección de las $\mathrm{UCl}$ que participaron en el grupo control e intervención no fueron aleatorizadas; las UCI de intervención y de control partían de una incidencia de BCVC aparentemente diferentes, siendo el doble en el grupo control; la incidencia de BCVC disminuyó también en las $\mathrm{UCl}$ de grupo control, posiblemente debido al conocimiento de las experiencias publicadas y del protocolo de intervención; no se compararon variables confundentes entre los pacientes del grupo intervención y control que podrían influir en la tasa de BCVC; no se documentaron los microorganismos responsables de la BCVC y al evitar la femoral pudo haber un cambio en la flora debido a que los microorganismos pueden ser diferentes según el acceso vascular ${ }^{24-27}$. Otra limitación del estudio piloto consistió en que el seguimiento de todas estas medidas preventivas fue de solo tres meses y no se podía saber la tendencia de la incidencia de BCVC. Sin embargo, durante la implantación del Proyecto Bacteriemia Zero a nivel nacional (1 de enero de 2009 hasta 30 de junio de 2010) se ha documentado una incidencia de BCVC de 3,05 episodios/1.000 días de CVC y en los últimos seis meses del proyecto estuvo en torno a 2,50 episodios/ 1.000 días de CVC (es decir, se consiguió el objetivo propuesto de bajar la incidencia de 4 episodios/1.000 días de $\mathrm{CVC}^{22}$ ).

Actualmente, se podría plantear la utilización de otras medidas preventivas como el uso de CVC o apósitos impregnados en determinadas situaciones. Estas medidas suponen un incremento del coste asistencial y debería seleccionarse apropiadamente sus indicaciones para optimizar la relación coste-beneficio.

Los CVC impregnados en antimicrobianos reducen la colonización microbiana en la superficie de sus paredes y potencialmente el riesgo de BCVC. El coste directo de estos CVC impregnados en antimicrobianos es bastante superior al de los CVC estándar; sin embargo, en varios metaanálisis se ha evidenciado que producen una disminución de la BCVC y de los costes asistenciales ${ }^{28-36}$. Los resultados de un estudio retrospectivo realizado por nuestro equipo sugieren que se podría optimizar la relación del coste-beneficio de estos CVC impregnados si se utilizaran en situaciones de mayor riesgo de BCVC, como en el acceso venoso femoral ${ }^{37}$.

Otra posible medida preventiva consistiría en la utilización de apósitos impregnados en gluconato de clorhexidina, los cuales reducen la colonización cutánea y potencialmente el riesgo de BCVC. El uso de estos apósitos ha disminuido la colonización de la punta del catéter en algunos estudios ${ }^{38-41}$ y en otros incluso la BCVC y los costes asistenciales ${ }^{42,43}$. Una limitación para el uso de apósitos impregnados en clorhexidina sería la aparición de dermatitis, sobre todo en pacientes menores de dos meses ${ }^{39}$.

En las guidelines de la Society for Healthcare Epidemiology of America/Infectious Diseases Society of America (SHEA/IDSA) para la prevención de la BCVC se propone la utilización de un CVC impregnado en pacientes adultos (nivel de recomendación A-I debido a la existencia de al menos un estudio controlado aleatorizado) o apósitos impregnados en clorhexidina en pacientes mayores de dos meses (nivel de recomendación B-I debido a la existencia de al menos un estudio bien diseñado, pero sin aleatorización) en las siguientes circunstancias ${ }^{44}: 1$ ) unidades hospitalarias o grupos de pacientes que tienen una incidencia de BCVC superior a la estimada a pesar de la cumplimentación de las medidas básicas para la prevención de BCVC; 2) pacientes que tienen limitaciones de accesos venosos y antecedentes personales de recurrente BCVC, y 3) pacientes con alto riesgo de secuelas debido a BCVC (ej., pacientes con implantación reciente de dispositivos intravasculares como válvulas cardíacas o prótesis aórticas). Aunque posteriormente a la publicación de estas guías se comunicaron dos estudios aleatorizados donde se objetivó una reducción de BCVC con los apósitos impregnados en clorhexidina ${ }^{42,43}$.

En el borrador de las guidelines de los Centers for Disease Control and Prevention (CDC) para la prevención de la BCVC se propone la utilización de un CVC impregnado en pacientes adultos (nivel de recomendación $\mathrm{A}-\mathrm{I}$ ) o apósitos impregnados en clorhexidina en pacientes mayores de dos meses (nivel de recomendación $\mathrm{B}-\mathrm{I}$ ) cuando las tasas se mantienen por encima de los objetivos propuestos por la institución después de implementar una comprensiva estrategia para reducir las tasas de BCVC (educación, utilizar máximas precauciones estériles y preparar la zona cutánea con clorhexidina al $2 \%)^{45}$. Aunque ya se analizaron los dos estudios aleatorizados donde se objetivó una reducción de BCVC con los apósitos impregnados en clorhexidina, no se estableció el nivel de recomendación A-I para el uso de apósitos impregnados ${ }^{42,43}$.

En mi opinión, se podría considerar el uso de catéteres impregnados $\mathrm{y} / \mathrm{o}$ apósitos impregnados en las siguientes circunstancias: canalización de accesos vasculares con mayor riesgo de bacteriemia, pacientes inmunodeprimidos y pacientes con alteraciones de la integridad cutánea. Según los datos de nuestra unidad, el número de episodios de bacteriemia relacionada con catéter vascular por 1.000 días de catéter según el sitio de acceso son los siguientes: 0 en arteria pedia $^{46}$ y $_{\text {braquial }}{ }^{47}, 0,25$ en arteria radial $^{48}, 0,9$ en catéteres venosos insertados periféricamente ${ }^{49}, 0,97$ en vena subclavia $^{50}, 1,2$ en vena yugular por acceso posterior ${ }^{51}$, 1,92 en arteria femoral ${ }^{48}, 4,8$ en vena yugular por acceso central ${ }^{52}, 5,1$ en vena subclavia con traqueostomía ${ }^{53}, 9,5$ en vena femoral ${ }^{54}$ y 21,64 en vena yugular central con traqueostomía ${ }^{55}$. Por lo tanto, la utilización de catéteres y/o apósitos impregnados presentaría una mejor relación coste/beneficio en la cateterización venosa yugular por acceso central con traqueostomía y en la venosa femoral. $Y$ aunque debe evitarse el acceso venoso femoral, podría contemplarse su utilización en determinados pacientes con un mayor riesgo de complicaciones mecánicas durante la canalización de vías venosas altas, como sería el caso de 
pacientes con alteraciones importantes del intercambio gaseoso, del estado hemodinámico, de la coagulación, del flujo arterial cerebral o de la caja torácica ${ }^{56}$.

Me gustaría concluir expresando mi felicitación a todas las personas que han estado implicadas en la realización del estudio piloto y del Proyecto Bacteriemia Zero por los resultados obtenidos en la reducción de la incidencia de BCVC.

\section{Bibliografía}

1. Vaqué J, Rosselló J, Arribas JL. Prevalence of nosocomial infections in Spain: EPINE study 1990-1997. EPINE Working Group. J Hosp Infect. 1999;43(Suppl):S105-11.

2. Vincent JL, Bihari DJ, Suter PM, Bruining HA, White J, Nicolas MH; and the EPIC International Advisory Committee. The prevalence of nosocomial infection in Intensive Care Units in Europe. JAMA. 1995;274:639-44.

3. Lorente L, León C. Cateterización venosa femoral: ¿realmente hay que evitarla? Med Intensiva. 2009;33:442-9.

4. Lorente L, Málaga J, Martín MM, Mora ML. Retirada accidental de catéteres. Med Intensiva. 2002;26:279-84.

5. Lorente L, Galván R, Martín MM, Mora ML. Incidencias de las complicaciones infecciosas en la cateterización intravascular. Med Intensiva. 2003;27:224-8.

6. Lorente L, Huidobro MS, Martín MM, Jiménez A, Mora ML. Accidental catheter removal in critically ill patients: a prospective and observational study. Crit Care. 2004;8:229-33.

7. Spengler RF, Greenough WB. Hospital costs and mortality attributed to nosocomial bacteremias. JAMA. 1978;240:2455-8.

8. Haley RW, Schaberg DR, Crossley KB, Von Allmen SD, McGowan JE. Extra charges and prolongation of hospitalization attributable to nosocomial infections: a prospective interhospital comparison. Am J Med. 1981;70:51-8.

9. Smith RL, Meixler SM, Simberkoff MS. Excess mortality in critically ill patients with nosocomial bloodstream infections. Chest. 1991;100:164-7.

10. Collignon PJ. Intravascular catheter associated sepsis: a common problem. The Australian Study on Intravascular Catheter Associated Sepsis. Med J Aust. 1994;161:374-8.

11. Pittet D, Tarara D, Wenzel R. Nosocomial bloodstream infection in critically ill patients. Excess length of stay, extra costs and attributable mortality. JAMA. 1994;271:1598-601.

12. Laupland KB, Lee $H$, Gregson DB, Manns BJ. Cost of intensive care unit-acquired bloodstream infections. J Hosp Infect. 2006;63:124-32.

13. Arnow PM, Quimosing EM, Beach M. Consequences of intravascular catheter sepsis. Clin Infect Dis. 1993;16:778-84.

14. Álvarez Lerma F, Olaechea Astigarraga P, Palomar Martínez M, Insausti Ordeñana J, López Pueyo MJ; Grupo de Estudio ENVINHELICS. Epidemiología de las bacteriemias primarias y relacionadas con catéteres vasculares en pacientes críticos ingresados en servicios de medicina intensiva. Med Intensiva. 2010;34: 437-45.

15. Álvarez-Lerma F, Palomar $M$, Olaechea $P$, Otal JJ, Insausti J, Cerdá E; Grupo de Estudio de Vigilacia de Infección Nosocomial en UCl. Estudio Nacional de Vigilancia de Infección Nosocomial en Unidades de Cuidados Intensivos. Informe evolutivo de los años 2003-2005. Med Intensiva. 2007;31:6-17.

16. Suetens C, Morales I, Savey A, Palomar M, Hiesmayr M, Lepape A, et al. European surveillance of ICU-acquired infections (HELICSICU): methods and main results. J Hosp Infect. 2007;65(Suppl 2): 171-3.

17. National Nosocomial Infections Surveillance System. National Nosocomial Infections Surveillance (NNIS) System Report, data summary from January 1992 through June 2004, issued October 2004. Am J Infect Control. 2004;32:470-85.

18. Edwards JR, Peterson KD, Mu Y, Banerjee S, Allen-Bridson K, Morrell G, et al. National Healthcare Safety Network (NHSN) report: data summary for 2006 through 2008, issued December 2009. Am J Infect Control. 2009;37:783-805.

19. Rosenthal VD, Maki DG, Jamulitrat S, Medeiros EA, Todi SK, Gómez DY, et al.; INICC Members. International Nosocomial Infection Control Consortium (INICC) report, data summary for 2003-2008, issued June 2009. Am J Infect Control. 2010;38. 95-104.e2.

20. Pronovost P, Needham D, Berenholtz S, Sinopoli D, Chu H, Cosgrove $S$, et al. An intervention to decrease catheter-related bloodstream infections in the ICU. N Engl J Med. 2006;355: 2725-32.

21. Warren DK, Cosgrove SE, Diekema DJ, Zuccotti G, Climo MW, Bolon MK, et al.; Prevention Epicenter Program. A multicenter intervention to prevent catheter-associated bloodstream infections. Infect Control Hosp Epidemiol. 2006;27:662-9.

22. Proyecto Bacteriemia Zero. Disponible en: http://hws.vhebron. net/bacteriemia-zero/bzero.asp.

23. Palomar M, Álvarez Lerma F, Alba Riera M, Leon Gil C, López Pueyo MJ, Díaz $C$, et al. Prevención de la bacteriemia relacionada con catéteres en $\mathrm{UCl}$ mediante una intervención multifactorial. Informe del estudio piloto. Med Intensiva. 2010;34:578-86.

24. Lorente L, Jiménez A, Iribarren JL, Jiménez JJ, Martín MM, Mora ML. The micro-organism responsible for central venous catheter related bloodstream infection depends on catheter site. Intensive Care Med. 2006;32:1449-50.

25. Lorente L, Jiménez A, Jiménez JJ, Iribarren JL, Martín MM, Mora ML. The catheter site influences in the micro-organism responsible of arterial catheter-related infection. Intensive Care Med. 2006;32:1919-20.

26. Lorente L, Jiménez A, Santana M, Iribarren JL, Jiménez JJ, Martín MM, et al. Microorganism responsibles for intravascular catheter-related bloodstream infection according to the catheter site. Crit Care Med. 2007;35:2424-7.

27. Lorente L, Jiménez A, Mora ML. Microorganism responsibles for femoral catheter-related bloodstream infection. Crit Care Med. 2008;36:657-8.

28. Hockenhull JC, Dwan K, Boland A, Smith G, Bagust A, Dündar Y, et al. The clinical effectiveness and cost-effectiveness of central venous catheters treated with anti-infective agents in preventing bloodstream infections: a systematic review and economic evaluation. Health Technol Assess. 2008;12:1-154.

29. Gilbert RE, Harden M. Effectiveness of impregnated central venous catheters for catheter related blood stream infection: a systematic review. Curr Opin Infect Dis. 2008;21:235-45.

30. Ramritu P, Halton K, Collignon P, Cook D, Fraenkel D, Battistutta D, et al. A systematic review comparing the relative effectiveness of antimicrobial-coated catheters in intensive care units. Am J Infect Control. 2008;36:104-17.

31. Marin MG, Lee JC, Skurnick JH. Prevention of nosocomial bloodstream infections: effectiveness of antimicrobial-impregnated and heparin-bonded central venous catheters. Crit Care Med. 2000;28:3332-8.

32. Pai MP, Pendland SL, Danziger LH. Antimicrobial-coated/bonded and -impregnated intravascular catheters. Ann Pharmacother. 2001;35:1255-63.

33. Saint S, Veenstra DL, Lipsky BA. The clinical and economic consequences of nosocomial central venous catheter-related infection: are antimicrobial catheters useful? Infect Control Hosp Epidemiol. 2000;21:375-80.

34. Veenstra DL, Saint S, Saha S, Lumley T, Sullivan SD. Efficacy of antiseptic-impregnated central venous catheters in preventing catheter-related bloodstream infection: a meta-analysis. JAMA. 1999;281:261-7. 
35. Falagas ME, Fragoulis K, Bliziotis IA, Chatzinikolaou I. Rifampicin-impregnated central venous catheters: a meta-analysis of randomized controlled trials. J Antimicrob Chemother. 2007;59: 359-69.

36. Casey AL, Mermel LA, Nightingale P, Elliott TS. Antimicrobial central venous catheters in adults: a systematic review and meta-analysis. Lancet Infect Dis. 2008;8:763-76.

37. Lorente L, Lecuona M, Ramos MJ, Jiménez A, Mora ML, Sierra A. The use of rifampicin-miconazole-impregnated catheters reduces the incidence of femoral and jugular catheter-related bacteremia. Clin Infect Dis. 2008;47:1171-5.

38. Hanazaki K, Shingu K, Adachi W, Miyazaki T, Amano J. Chlorhexidine dressing for reduction in microbial colonization of the skin with central venous catheters: a prospective randomized controlled trial. J Hosp Infect. 1999;42:165-8.

39. Garland JS, Alex CP, Mueller CD, Otten D, Shivpuri C, Harris MC, et al. A randomized trial comparing povidone-iodine to a chlorhexidine gluconate-impregnated dressing for prevention of central venous catheter infections in neonates. Pediatrics. 2001;107:1431-6.

40. Chambers ST, Sanders J, Patton WN, Ganly P, Birch M, Crump JA, et al. Reduction of exit-site infections of tunnelled intravascular catheters among neutropenic patients by sustainedrelease chlorhexidine dressings: results from a prospective randomized controlled trial. J Hosp Infect. 2005;61:53-61.

41. Levy I, Katz J, Solter E, Samra Z, Vidne B, Birk E, et al. Chlorhexidine-impregnated dressing for prevention of colonization of central venous catheters in infants and children: a randomized controlled study. Pediatr Infect Dis J. 2005;24:676-9.

42. Ruschulte $H$, Franke M, Gastmeier P, Zenz S, Mahr KH, Buchholz S, et al. Prevention of central venous catheter related infections with chlorhexidine gluconate impregnated wound dressings: a randomized controlled trial. Ann Hematol. 2009;88:267-72.

43. Timsit JF, Schwebel C, Bouadma L, Geffroy A, Garrouste-Orgeas M, Pease S, et al.; Dressing Study Group. Chlorhexidine-impregnated sponges and less frequent dressing changes for prevention of catheter-related infections in critically ill adults: a randomized controlled trial. JAMA. 2009;301:1231-41.

44. Marschall J, Mermel LA, Classen D, Arias KM, Podgorny K, Anderson DJ, et al. Practice Recommendation of Society for Healthcare Epidemiology of America/Infectious Diseases Society of America (SHEA/IDSA). Strategies to prevent central line-associated bloodstream infections in acute care hospitals. Infect Control Hosp Epidemiol. 2008;29(Suppl 1):S22-30.
45. O'Grady NP, Alexander M, Burns LA, Dellinger EP, Garland J, Heard SO, et al; Guidelines for the prevention of intravascular catheter-related infections: SCCM, IDSA, SHEA, ATS, CDC. Disponible en: http://www.cdc.gov/ncpdcid/pdf/Draft_BSI_gui deline_v15_2FR.pdf.

46. Lorente L, Jiménez A, Jiménez JJ, Iribarren JL, Martínez J, Naranjo C, et al. Higher arterial catheter-related infection rates in femoral than in dorsalis pedis access. J Hosp Infect. 2010;74:365-9.

47. Lorente L, Jiménez A, Martín MM, Jiménez JJ, Iribarren JL, Mora ML. Lower arterial catheter-related infection in brachial than in femoral access. Am J Infect Control. 2010 [Epub ahead of print].

48. Lorente L, Santacreu R, Martín MM, Jiménez A, Mora ML. Arterial catheter-related infection of 2,949 catheters. Crit Care. 2006;10:R38.

49. Lorente L, Villegas J, Martín MM, Jiménez A, Mora ML. Catheterrelated infection in critically ill patients. Intensive Care Med. 2004;30:1681-4.

50. Lorente L, Henry C, Martín MM, Jiménez A, Mora ML. Central venous catheter-related infection in a prospective and observational study of 2,595 catheters. Crit Care. 2005;9:631-5.

51. Lorente L, Jiménez A, Galván R, García C, Castedo J, Martín MM, et al. Equivalence of posterior internal jugular and subclavian accesses in the incidence of central venous catheter related bacteremia. Intensive Care Med. 2007;33:2230-1.

52. Lorente L, Jiménez A, Castedo J, Galván R, García C, Martín MM, et al. Internal jugular venous catheter-related bacteremia according to central and posterior accesses. Intensive Care Medicine. 2007;33:1071-5.

53. Lorente L, Jiménez A, Martín MM, Castedo J, Galván R, García C, et al. Influence of tracheostomy on the incidence of central venous catheter-related bacteremia. Eur J Clin Microbiol Infect Dis. 2009;28:1141-5.

54. Lorente L, Jiménez A, García C, Galván R, Castedo J, Martín MM, et al. Catheter-related bacteremia from femoral and central internal jugular venous access. Eur J Clin Microbiol Infect Dis. 2008;27:867-71.

55. Lorente L, Jiménez A, Naranjo C, Martínez J, Iribarren JL, Jiménez JJ, et al. Higher incidence of catheter-related bacteremia in Jugular site with tracheostomy than in femoral site. Infect Control Hosp Epidemiol. 2010;31:311-3.

56. Lorente L, León C. Cateterización venosa femoral: ¿una opción en determinados pacientes? Med Intensiva. 2010;34:221-3. 